\title{
Research on the Neurobiology of COVID-19
}

\author{
Yilun Tong ${ }^{1}$ \\ ${ }^{1}$ The Stony Brook School, Stony Brook, NY 11790, USA
}

\begin{abstract}
More studies have shown the neurological manifestations of the novel corona virus (COVID-19) and have inferred the molecular mechanism by which it invades the nervous system. The neurological aspect of the COVID-19 pandemic has been differently interpreted and dealt with in different parts of the world. To review the neurological manifestations and the neurovirulent mechanism by which $\mathrm{CoV}$ attacks the human nervous system and to examine different perspectives on this very same topic, the research on PubMed and ScienceDirect is conducted. The mechanisms that CoV enter and attack the nervous system and the subsequent neurologic manifestations have been proposed and now seems quite clear. However, more studies have to be done directly on the effect of COVID-19 on the CNS as well as the PNS.
\end{abstract}

\section{Introduction}

The novel COVID-19 was recognized as a pandemic by WHO in April 2020. As of July 2020, there have been approximately 16.3 million confirmed cases of the novel corona virus globally [1]. In fact, in the beginning of the 21 st century, there have been three major outbreaks of respiratory infections: the severe acute respiratory syndrome (SARS) that took place from 2002 to 2003 by SARS-CoV, the Middle East Respiratory Syndrome (MERS) by MERS-CoV, and the ongoing one caused by SARS-CoV2. All three CoV viruses firstly infect lower animals and then spread among human population. The pathophysiological symptoms that follows the infection include coughing, sore throat, muscle pain, etc. [2]

Patients may show neurologic symptoms as well as a result of the viral infection. This includes a change in mental status, seizure, and confusion [3]. A considerable number of literature has shown some of the neurological manifestations of COVID-19, and common grounds have been established regarding the potential mechanisms that SARS-CoV2 may have played in invading, particularly, the central nervous system. Two major types of neurovirulent mechanisms will be discussed in this review.

There are yet varying perspectives on the interpretation of the neurological complication of COVID-19 by SARS-CoV2, which will be compiled and discussed later. This article will provide another brief overview on the researches and reports that have been done regarding the neural manifestations and neuroinvasive mechanism of SARS-CoV2. In addition, the article will explain some of the differences in terms of interpretations across countries.

\section{Common grounds established: an overview}

The vast majority of the searched studies have an unanimous conclusion on the neurological symptoms of COVID-19 infection and the molecular neurovirulence of the corona viruses. The article presents a brief overview of both.

\subsection{Reported cases and neurological manifestations of COVID-19}

Many confirmed COVID-19 cases have been reported from different nations since the early stage of the pandemic. As mentioned before, whereas most reported cases show symptoms involving multi-organ system dysregulations[2] and respiratory syndromes[4]. Neurologic symptoms are shown by a significant number of the reported cases as well [12]. A few studies on confirmed COVID case series and some of those neurological manifestations of COVID-19 infection will be identified and discussed here in this section.

In a study recently conducted by Mao et al. in Wuhan, China, 214 patients, 88 of which being identified as severely cases. Neurologic symptoms of COVID-19 infection were reported after a retrospective chart review. In total, $45.5 \%$ of the patients are observed to show neurologic symptoms, most of which are central nervous system damage involving dizziness, headache, and impaired consciousness; And $8.9 \%$ of the 214 patients presented peripheral nervous system impaiement [13] such as hypogeusia and hyposmia [5]. The study conducted by Helm et al. in France, too, indicated the neurologic manifestations of $2019-\mathrm{nCoV}$ infection. In their report, $84 \%$ of the patients experienced neurological symptoms. $69 \%$ of the patients experienced agitation and 
$65 \%$ of experienced delirium as documented by CAMICU [6]. Below is a more complete summary of these two case reports.

There are reported individual studies from all over the world, though not published. In Japan, a COVID-19 patient who experienced seizure after severe brain edema was reported [7]. There have been multiple reports of COVID-19-associated Guillain-Barré syndromes [8] as well as encephalitis [9] It is clear that neuropathy/encephalopathy are indeed closely associated with SARS-CoV2 infection.

Table 1. Neurologic manifestations in two most cited case series [10]

\begin{tabular}{|c|c|c|c|}
\hline \multicolumn{4}{|l|}{ se } \\
\hline & Mao et al., 2020 & Helms et al., 2020 & Total \\
\hline Method & Retrospective chart review & Observational study & \\
\hline Number of Patients & 214 & 58 & 273 \\
\hline Median Age & 58.7 & 63 & \\
\hline Neurologic Symptoms & $45.5 \%$ & $83 \%$ & $53.3 \%$ \\
\hline Dizziness & $19.3 \%$ & NR & \\
\hline Headache & $17.1 \%$ & NR & \\
\hline Agitation & NR & $69 \%$ & \\
\hline Delirium documented by CAM-ICU & NR & $65 \%$ & \\
\hline Impaired consciousness & $14.8 \%$ & NR & \\
\hline Hypogeusia & $5.6 \%$ & NR & \\
\hline Hyposmia & $5.1 \%$ & NR & \\
\hline Ischemic stroke & $5.7 \%$ & $23 \%(3 / 13)$ & $5.6 \%$ \\
\hline
\end{tabular}

* CAM-ICU: Confusion assessment method in Intensive care unit

\subsection{Mechanisms of SARS-CoV2/CoV invasion}

Given the neurological manifestation associated with SARS-CoV2 and other corona viruses, studies have been done on how exactly different viruses in the CoV family (particularly SARS-CoV1, MERS, and SARS-CoV2) might attack the human nervous system on the molecular and cellular level. Organizing findings and hypotheses from various studies, mechanisms by which SARS-CoV2 invade the nervous system, mostly derived from those of SARS-CoV1 can be divided into two categories: direct and indirect.[11]

The Direct's as self-explanatory as the name "direct infection" is, it stands for the process in which the viruses directly invade the nervous system. This is supported by the discovery of genetic materials and protein components of the viruses directly in the tissues in the nervous system. CoV might directly attack the nervous system in two distinct ways. The first way is through neuronal pathways, by which the olfactory bulb, due to its distinct anatomy, acts as a channel between the nasal epithelium and the central nervous system. The second mechanism, though supported by only a few studies, is viral infection through blood circulation [4]. Another direct neuroinvasive mechanism is through angiotens in converting enzyme 2 (ACE2). ACE2 receptors to which $\mathrm{CoV}$ spike proteins target, are found mainly on lung alveolar epithelium, yet they are found, too, in the central nervous system, which indicates possible neurotropism [11], another way by which $\mathrm{CoV}$ attack our nervous system directly. The viruses may enter the central nervous system by damaging the bloodbrain barrier and attacking the vascular system [4].

The Indirect's As mentioned just now, ACE2 receptors are present primarily on the lung epithelium, suggesting an indirect pathway of viral attack. CoV viruses may bind to these receptors, which in turn raises blood pressure putting the brain at a higher risk of cerebral hemorrhage. Another indirect mechanism is injury by hypoxia. The proliferation of $\mathrm{CoV}$ in the lung causes edema and inflammation, which in turn results in disruptions in gas exchange and CNS hypoxia[5]. Immune injury is yet another mechanism. Activation of the immune system will in turn cause inflammation and brain damage. Below is a succinct summary of different types of neuroinvasion of $\mathrm{CoV}$ : 


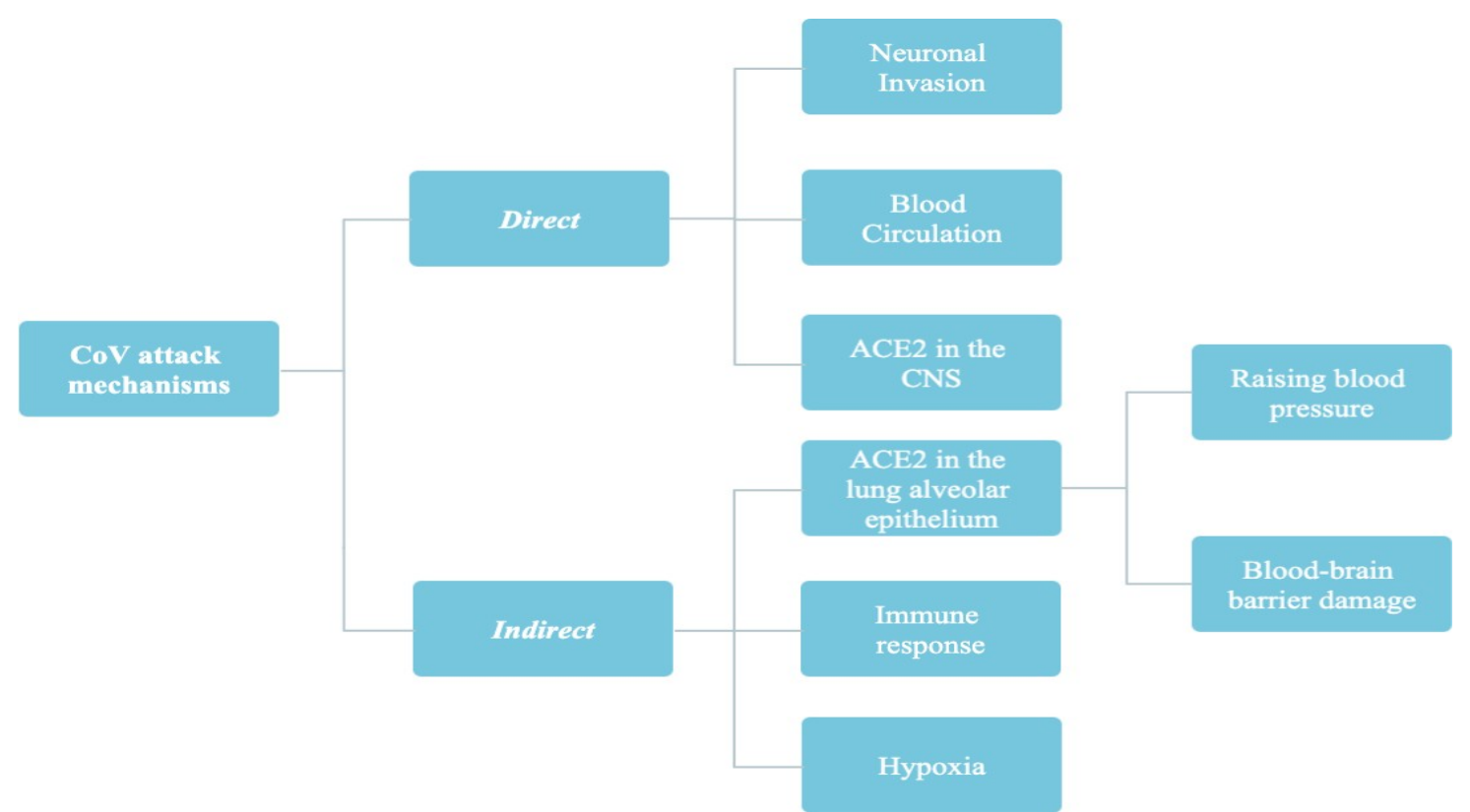

Figure 1. Different mechanisms by which CoV (presumably SARS-CoV2) attack nervous system [2]

It is a unanimous assumption, though not supported by much evidence, on how SARS-CoV2 invade our nerves, as well as what the neurologic manifestations of COVID-19 are.

\section{Discussion}

Although many common grounds, discussed in the last section, have been established on this very topic. It is easy to observe numerous differences between researches and studies from all over the world. Here the article argues that the discrepancies come from two aspects: the varying physiological features of people at different ages, and the differences in what to emphasize in different cultures as to how to deal with neurologic symptoms. There are limitation of the current studies, which will be elaborated in this section as well.

\subsection{Different perspectives}

The Ages In the studies done by Mao et al. and Helms et al., several neurologic manifestations are discussed. $45.5 \%$ and $84 \%$ of the studies patients, respectively, showed neurologic symptoms in these two studies. Yet many other studies documented few patients with neurologic manifestations. This is because of the median age reported in Mao et al. and Helms et al.'s studies: 58.7 and 63, respectively, both are high. One can postulate that neurologic symptoms, along with vulnerability to $\mathrm{CoV}$ increases when one gets older. More case series studying younger groups of people are required in order to find out a correlation between the severity of neurological manifestations and age.

The Focuses in the same studies[6,10], one can easily observe the differences of the neurologic symptoms reported. Mao et al. from China mainly reported neural manifestations associated with physical features, such as dizziness and hypogeusia, whereas Helms et al. from France mainly reported manifestations more on a cognitive and neurological basis, like agitation and delirium (Chart 1). This difference is not uncommon: It is possible to assume that the definition of a neurological manifestation may vary across countries. For example, a neural manifestation may be described as a symptom that can be shown physically under the Chinese medical care system, while it may be described as cognitive and mental impairment in France. Therefore, whereas observable and instant treatments are the main emphasis in the Chinese medical care system, long-lasting, cognitive illnesses could be the main focus of the French and even some other western countries. It is wise if medical carers from the correspondent countries are able to convene and corporate allowing the exchange of perspectives.

\subsection{Other limitations}

Though the neurologic influence of the virus is studies, but limitations still exist is undoubtable. Many of these studies are not solely studying the neurologic symptoms[3], indicating a limitation in these current studies. It is easy to infer that the neurological aspect of $\mathrm{CoV}$ infection still is not the main focus of the current field. Yet a few studies have already concluded that those severely infected show more neurologic symptoms and fewer respiratory ones. Thus, neurologic manifestations of COVID-19 and other CoVs are essential to understand how the viruses might affect a person as a whole. Further studies are required, only directly studying the neural manifestations and complications. In addition, the mechanism that SARS-CoV2 attack the human nervous system is proposed solely by referencing that of SARSCoV1 and the fact that both viruses behave in similar fashions [3] Further studies directly on SARS-CoV2 are required, too. Furthermore, not a good number of studies have been done using EEG or CSF analysis, indicating a potential inaccuracy in the neurologic manifestations reported. 


\section{Conclusion}

Studies have been done on the neurovirulence of coronaviruse, indicating that potential neurologic manifestations may include both peripheral and central nervous system impairment. The molecular and cellular mechanisms have been directly and indirectly postulated. The viruses enter and affect the nervous system. However, there still are differences as to what exactly is a neurologic manifestation and the severity of neurological symptoms in patients, and can potentially be explained as a product of both age difference and different subjects of focus. Yet more studies are requires to directly and solely study the effect of SARS-CoV2 (COVID-19) on the human nervous system, and technologies such as CSF analysis and EEG are also required to get more accurate results.

\section{ACKNOWLEGEMENT}

I would like to give thanks to Professor Christopher Deppmann, who offered me new perspectives as well as feedbacks during the writing process of this article. I would also like to give special thanks to my high school psychology instructor, who offered advice on this article. Without their insights, this paper can hardly be finished.

\section{References}

1. https://www.who.int/emergencies/diseases/novelcoronavirus-2019

2. Cataldi M, Pignataro G, Taglialatela M. Neurobiology of coronaviruses: Potential relevance for COVID-19. Neurobiology of Disease 2020.

3. Asadi-Pooya A, Simani L, et al. Central nervous system manifestations of COVID19: A systematic review. Journal of the Neurological Sciences 2020.

4. Wu Y, Xu X, Chen Z, Duan J, Hashimoto K, Yang L, Liu C, Yang C. Nervous system involvement after infection with COVID-19 and other coronaviruses. Brain, Behavior, and Immunity 2020.

5. Ahmad I, Rathore F. Neurological manifestations and complications of COVID-19: A literature review. Journal of the Neurological Sciences 2020.

6. Helms J, Kremer S, Merdji H, Clere-Jehl R, Schenck M, Kummerlen C, et al. Neu- rologic Features in Severe SARS-CoV-2 Infection. N Engl J Med 2020.

7. Kadano Y, Nakamura Y, Ogawa Y, Yamamoto S, Kajikawa R, Nakajima Y, Mat- sumoto M, Kishima H. A case of COVID-19 infection presenting with a seizure following severe brain edema. Seizure 2020.

8. Gupta A, Paliwal K., Gard R. Is COVID-19-related Guillain-Barré syndrome differ- ent? Brain, Behavior, and Immunity 2020.

9. De Felice F, Tovar-Moll F, Moll J, Munoz D, Ferreira S. Severe Acute Respiratory Syndrome Coronavirus 2 (SARS-CoV-2) and the Central Nervous System. Cell Press Re- views 2020.
10. MaoL, JinH, WangM, HuY, ChenS, HeQ, ChangJ, HongC, ZhouY, WangD, Miao X, Li Y, Hu B. Neurologic manifestations of hospitalized patients with coronavirus disease 2019 in Wuhan, China. JAMA Neurol 2020.

11. Werner C, Scullen T, Mathkour M, Zeoli T, Beighley A, Kilgore M, Carr C et al. Neurological Impact of Coronavirus Disease of 2019: Practical Considerations for the Neuroscience Community. World Neurosurgery 2020.

12. Matias-Guiu J, Gomez-Pinedo U, MonteroEscribano P, Gomez-Iglesias P, Porta- Etessam J, Matias-Guiu J.A. Should we expect neurological symptoms in the SARS-CoV-2 epidemic. Neurologia 2020.

13. Montalvan V, Lee J, Bueso T, De Toledo J, Rivas K. Neurological manifestations of COVID-19 and other coronavirus infections: A systematic review. Clinical Neurology and Neurosurgery 2020. 